\title{
Antioxidant protein peroxiredoxin 6 suppresses the vascular inflammation, oxidative stress and endothelial dysfunction in angiotensin II-induced endotheliocyte
}

\author{
Dai-Xu Li ${ }^{1}$, Wei Chen ${ }^{2}$, Ying-Lei Jiang ${ }^{3}$, Jing-Qin $\mathrm{Ni}^{2}$ and $\mathrm{Lin}^{2}{ }^{2}$ \\ ${ }^{1}$ The First Department of Cardiovascular Medicine, The Third Affiliated Hospital of Shandong First Medical University and \\ The Fourth People's Hospital of Jinan, Jinan, Shandong, China \\ ${ }^{2}$ Department of Cardiovascular Medicine, The Third Affiliated Hospital of Shandong First Medical University, Jinan, \\ Shandong, China \\ ${ }^{3}$ Jinan Stomatological Hospital Shungeng Hospital Area, Jinan, Shandong, China
}

\begin{abstract}
Cardiovascular disease (CVD) states are associated with endothelial dysfunction (ED) and increased production of ROS in endothelial cells. The present study aimed to explore the protective effects of antioxidant protein peroxiredoxin 6 (PRDX6) on angiotensin II (AngII)-induced human umbilical vein endothelial cell (HUVEC) dysfunction. To investigate cell viability, levels of inflammatory molecules and proteins were assayed using the CCK- 8 assay and evaluated by ELISA and Western blot. NO and ROS levels were determined by Griess assay and the fluorescent probe DCFH-DA. Cell migration capacity was assessed by Transwell assay. AngII decreased cell viability and PRDX6, upregulated the expression levels of TNF- $\alpha$, IL-6, IL-1 $\beta$, LDH and MDA, stimulated ROS production, and reduced NO synthase, the expressions of eNOS, MnSOD, ICAM-1, VCAM-1, and activated the MAPK family of signaling proteins. However, the stimulatory effects of AngII on HUVECs were remarkably suppressed by PRDX6. Furthermore, mercaptosuccinate (MS; PRDX6 inhibitor) had similar effects as AngII in aggravating HUVECs damage. Conversely, these adverse events caused by AngII and MS were obviously reversed by ML3404 and SP600125. The present study indicated that PRDX6 overexpression inactivated p38 MAPK and JNK pathway through decrease AngII-induced inflammation, oxidative stress and endothelial dysfunction leading to attenuation of endothelial cell damage.
\end{abstract}

Key words: Peroxiredoxin 6 - Cardiovascular disease - Oxidative stress - Inflammation Angiotensin II

\section{Introduction}

Oxidative stress, a term defining states characterized by elevated ROS levels, and ROS as an important biomolecular factor, plays a key role in inducing endothelial cells damage and causing endothelial dysfunction which participated

Correspondence to: Lin Lu, Department of Cardiovascular Medicine, The Third Affiliated Hospital of Shandong First Medical University (Affiliated Hospital of Shandong Academy of Medical Sciences), No. 38 Wuying Mountain Road, Tianqiao district, Jinan, Shandong, 250031, China

E-mail: LLin901@163.com in the development of numerous cardiovascular disease (CVD) (Bendall et al. 2007). Recent studies have shown that ROS plays a pathophysiological role in endothelial dysfunction (ED), inflammation, migration, angiogenesis underlying cardiovascular in hypertension and diabetes (Paravicini and Touyz 2008), which is associated with reduced NO bioavailability (Cai and Harrison 2000). Generally, oxidative stress causes the damage of proteins and DNA, resulting in cellular dysfunction (Sun et al. 2012). Additionally, oxidative modification of proteins may result in the formation of nitro tyrosine, which represents a powerful and autonomous marker of cardiovascular diseases (Shishehbor et al. 2003). Therefore, the develop- 
ment of antioxidant proteins may be a new target for the treatment of CVD.

ROS, a chemically reactive molecules containing oxygen, serves as intracellular signaling pathways such as mitogenactivated protein kinases (MAPKs) involved in cell growth, gene expression and apoptosis (Nathan 2003). Peroxiredoxins (PRDXs) are antioxidant enzymes that primarily function as antioxidants to scavenge peroxide in biological system (Patel and Chatterjee 2019). ROS can be scavenged by peroxidase and PRDXs represent a superfamily of non-seleno peroxidase (Rhee et al. 2001). Unlike the other members of PRDXs family with two reactive cysteines, PRDX6 has a single redox-active cysteine residue and is distributed across various cellular sites of ROS production (Rhee et al. 2001, 2005). Yang et al. (2011) reported that PRDX6 deficiency exacerbates lipopolysaccharide (LPS)-induced acute lung injury in mice through increasing oxidative stress and Lee et al. (2017) hold that PRDX6 overexpression attenuates LPSinduced acute kidney injury, indicating that PRDX6 may play protective role in LPS-induced tissue injury. In addition, as an antioxidant, PRDX6 participated in the generation of ROS, specifically in endothelial cells (Chatterjee et al. 2011). ROS production occurs via PRDX6-phospholipase A2 (PLA2) activity, lack of PRDX6 leads to compromised ROS production with mechanotransduction (Chatterjee et al. 2011). Thus, PRDX6 performs a role in antioxidant defense by scavenge of hydrogen peroxide.

Studies using various cell lines showed that overexpression of PRDX6 increases resistance to experimental oxidative stress (Wang et al. 2008). AngII-dependent hypertension is an endogenous agonist that raises blood pressure and is particularly sensitive to NADPH oxidase-derived ROS. A previous study showed that AngII activates the NADPH oxidase (NOX2) pathway on endothelial cells in vitro (Vazquez-Medina et al. 2016). The ROS family comprises many molecules that influence vascular cell growth, migration, proliferation, inactivation of NO, stimulation of many kinases (p38, JNK) and proinflammatory genes (TNF- $\alpha$, IL-1 $\beta$, IL-6) (Cai 2005; Mueller et al. 2005; Touyz 2005; Harrison et al. 2006). Importantly, many of these actions are associated with pathological changes observed in CVD.

As a crucial factor of endothelial dysfunction, AngII participates in endothelial oxidative damage and inflammation, which is present in all CVD. It has previously been demonstrated that PRDX6 exerts cytoprotective effects against AngII-induced in pulmonary microvascular endothelial cells via antioxidant defense mechanisms (Vazquez-Medina et al. 2016). However, to the best of our knowledge, no previous studies have verified whether PRDX6 has any effect on AngII-induced vascular inflammation, oxidative stress and endothelial dysfunction in HUVECs. In the present study, HUVECs were treated with AngII to establish a cellular model in vitro, and the effects of AngII on oxidative stress were detected. Furthermore, the protective effects of PRDX6 on AngII-induced HUVECs were investigated. The effects of PRDX6 on AngII-induced ROS accumulation, inflammation, oxidative stress and endothelial dysfunction, which are important processes in the development of CVD, were also determined.

\section{Materials and Methods}

\section{HUVECs culture and treatments}

HUVECs were purchased from the Shanghai Institute for Biological Sciences, Chinese Academy of Sciences (Shanghai, China), and cultured in high Dulbecco's modified eagle medium (DMEM), supplemented with $10 \%$ fetal bovine serum (FBS), in incubator at $37^{\circ} \mathrm{C}$ with $5 \% \mathrm{CO}_{2}$. HUVECs $\left(1 \times 10^{5}\right.$ cells/per well) were treated with different concentrations of AngII $\left(10^{-5}, 10^{-6}, 10^{-7}, 10^{-8} \mathrm{M}\right)$ (Sigma, USA) for $48 \mathrm{~h}$. The medium was not changed during culture and treatment. The cells in control group were cultured in vehicle that is composed of 98\% DMEM and 2\% DMSO. AngII was dissolved in vehicle.

\section{Cell viability assay}

After treatment with AngII, HUVECs (80-90\% confluence) were seeded in a 96-well plate and $10 \mu \mathrm{l}$ of CCK-8 (Dojindo China Co., Ltd.; Shanghai, China) was then added into each well with $100 \mu \mathrm{l}$ medium for 1 -h incubation. Cell viability was determined at $450 \mathrm{~nm}$ by using a microplate reader. The percentage cell survival was determined by comparing the average absorbance of the treated cells to control cells. Meanwhile, the PRDX6 expression in HUVECs after treated with different concentrations of AngII were detected using Western blot as described in detail later. Based on the results, AngII of the trough value $\left(10^{-6} \mathrm{M}\right)$ accompanying by the $\geq 50 \%$ cell activity and a significant decrease in PRDX6 expression was selected for the following experimental study.

\section{Cell transfections and grouping}

HUVECs (80-90\% confluence) cells were transfected with plasmid containing pcDNA specific for PRDX6 (pcDNAPRDX6) and the scramble negative control (pcDNA-NC) using Lipofectamine 3000 (Invitrogen, USA) to overexpress the expression of PRDX6. Thereafter, HUVECs were harvested and used for the following experiments and divided into four groups: i) Control; ii) AngII, consisting of $1 \times 10^{5}$ HUVECs treated with $10^{-6} \mathrm{M}$ AngII for $48 \mathrm{~h}$; iii) AngII+pcDNA-NC, consisting of $1 \times 10^{5}$ HUVECs transfected with pcDNANC plasmids after treated with $10^{-6} \mathrm{M}$ AngII for $48 \mathrm{~h}$; 
iv) AngII+pcDNA-PRDX6, consisting of $1 \times 10^{5}$ HUVECs transfected with pcDNA-PRDX6 plasmids after treated with $10^{-6} \mathrm{M}$ AngII for $48 \mathrm{~h}$. Thereafter, the HUVECs were harvested and used for the following experiments.

Subsequently, for reverse validation studies, HUVECs were pretreated with p38 MAPK inhibitor ML3404 $(5 \mu \mathrm{M}$, Cat. No. 506121; Calbiochem) or JNK inhibitor SP600125 (10 $\mu \mathrm{M}$, Cat. No. 420119; Calbiochem) for $30 \mathrm{~min}$ before treated with PRDX6 inhibitor (Mercaptosuccinate, MS, $20 \mu \mathrm{M}$ ) (Sigma, USA) for $2 \mathrm{~h}$. HUVECs were then exposed to AngII $\left(10^{-6} \mathrm{M}\right)$ for $48 \mathrm{~h}$. The medium was not changed during culture and treatment. The experimental groups were as follows: Control, AngII, AngII+MS, AngII+MS+ML3404 and AngII+MS+SP600125.

\section{Enzyme-linked immunosorbent assay}

The levels of TNF- $\alpha$, IL- 6 and IL- $1 \beta$ in HUVECs were assayed using ELISA kits following the manufacturer's instructions. Briefly, after different treatments, HUVECs culture supernatants were collected. $100 \mu \mathrm{l}$ of the supernatants was added to the $96-$ well plate coated by special antibody, and incubated with Enzyme conjugation solution at $37^{\circ} \mathrm{C}$ for $60 \mathrm{~min}$. After washing the plates for 5 times, substrate I (DH2) and substrate II (3, 3', 5, 5'-Tetramethylbenzidine) were incubated at room temperature for $15 \mathrm{~min}$ in darkness respectively. The OD value was read at $450 \mathrm{~nm}$ using a microplate reader to evaluate the expression levels of TNF- $\alpha$, IL- 6 and IL- $1 \beta$.

\section{Measurement of intracellular ROS generation}

Intracellular ROS generation was tested by the oxidative conversion of cell-permeable oxidation of 2',7'-dichlorodihydrofluorescein diacetate (DCFH-DA) to fluorescent DCF. After receiving different treatments, $10 \mu \mathrm{M}$ DCFH-DA solution in serum-free DMEM was added to the wells, and then HUVECs were incubated for a further $30 \mathrm{~min}$ at $37^{\circ} \mathrm{C}$ in an incubator. After that, HUVECs were washed 5 times prior to the detection of DCF fluorescence over the entire field of vision using a fluorescence microscope connected to an imaging system (IX51, Olympus, Japan) with the excitation wavelength $488 \mathrm{~nm}$. The mean fluorescence intensity (MFI) of ROS from 5 random fields was measured using Image J 1.410 software (National Institutes of Health, USA) and the MFI was used to represent the amount of ROS. Magnification, $\times 100$.

\section{Measurement of antioxidant enzymes activities}

After various treatments, HUVECs were washed, collected and broken by ultrasound, and then centrifuged at 12,000 $\times g$ at $4^{\circ} \mathrm{C}$ for $5 \mathrm{~min}$. The supernatants were used to detect the activities of ROS and LDH and the concentration of MDA in the HUVECs according to the instructions of assay kits (Jiancheng Bioengineering Institute, Nanjing, PR China). The activities of antioxidant enzymes and MDA content were expressed as the percentage of the control value.

\section{Measurement of NO production}

NO has an extremely short half-life and quickly metabolizes to nitrate and nitrite. Thus, the level of nitrite in the supernatant collected from confluent treated cells was assayed by Griess method using a nitrate/nitrite colorimetric assay kit (R\&D Systems, Minneapolis, MN, USA) to represent NO production. Briefly, HUVECs supernatant was cultured with Griess reagent (Sigma) at room temperature for $5 \mathrm{~min}$ and the values of optical density were measured at $540 \mathrm{~nm}$ under a microplate reader. Sodium nitrite was used as a standard.

\section{Western blot assay}

After various treatments, HUVECs were lysed with ice-cold RIPA Lysis Buffer (Beyotime), which was incubated at $4^{\circ} \mathrm{C}$ for $30 \mathrm{~min}$ and centrifuged at $12,000 \times g$ for $20 \mathrm{~min}$ at $4^{\circ} \mathrm{C}$. Total protein concentrations were measured using bicinchoninic acid (BCA) method for protein quantification. Equal amount $(40 \mu \mathrm{g})$ of protein samples were fractionated by sodium dodecyl sulfate polyacrylamide gel electrophoresis (SDS-PAGE), and then electro-transferred onto polyvinylidene fluoride (PVDF) membranes (Millipore, USA). Membranes were blocked with $1 \times$ Tris-buffered saline-tween (TBST) containing 5\% nonfat dried milk at room temperature for $1 \mathrm{~h}$ and then were incubated at $4^{\circ} \mathrm{C}$ overnight with the following primary antibodies: Antibodies for PRDX6 (\#95336), MnSOD (\#13141), eNOS (Ser1177) (\#9570) were purchased from Cell Signaling Technology (USA), and ICAM-1 (sc-7891), VCAM-1 (sc-13610), p-p38 (sc-166182), p38 (sc-7972), p-JNK (sc-6254), JNK (sc-7345), ERK1/2 (sc-514302), p-ERK1/2 (sc-81492) and GAPDH (sc-137179) were obtained from Santa Cruz Biotechnology (Santa Cruz, CA, USA). Then, membranes were incubated with the corresponding secondary antibodies of HRP-conjugated goat anti-rabbit or anti-mouse (Santa Cruz Biotechnology Inc., USA) for $2 \mathrm{~h}$ at room temperature. The protein bands were visualized with enhanced chemiluminescence (ECL) detection kits and analyzed with Image J software to obtain gray values of the bands. GAPDH was used as an internal reference. The relative changes of proteins' content were quantified using the ratio of gray value of target protein to GAPDH.

\section{Transwell migration assay}

A total of $1 \times 10^{5}$ cells of HUVECs cultured in $100 \mu$ DMEM were placed on the upper chamber of a 24-well Transwell 
chamber ( $8 \mu \mathrm{m}$ pore size, BD, USA), while the lower chamber was filled with $300 \mu \mathrm{l}$ DMEM. After the system was incubated for $24 \mathrm{~h}$, the cells on the inner surface of upper chamber were scraped with a cotton swab, and the cells on the lower chamber were fixed with $4 \%$ paraformaldehyde for $10 \mathrm{~min}$. After fixation, the cells were stained with $0.1 \%$ crystal violet for $15 \mathrm{~min}$, and washed excessive dye with PBS and dried. Then, the migrated cells were imaged and counted under an optical microscope.

\section{Statistical analysis}

Statistical analysis was performed using SPSS 23 software. All the experiments were repeated independently more than three times. Data were expressed as mean \pm standard deviation (SD). If data followed a normal distribution, $t$-test was performed between two groups. The differences among groups were evaluated using one-way analysis of variance. $p<0.05$ was considered statistically significant.

\section{Results}

\section{Cytotoxicity increased and expression of PRDX6 decreased in AngII-induced HUVECs}

As presented in Fig. 1A, compared to untreated HUVECs, cell viability significantly decreased with the increase of dosage of AngII $\left(10^{-5}, 10^{-6}, 10^{-7}, 10^{-8} \mathrm{M}\right)$. Meanwhile, the mRNA level of PRDX6 was reduced obviously in AngII-
A

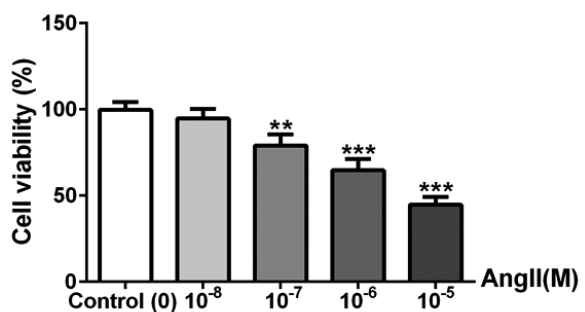

C

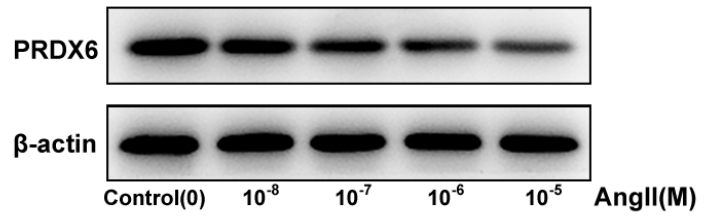

D

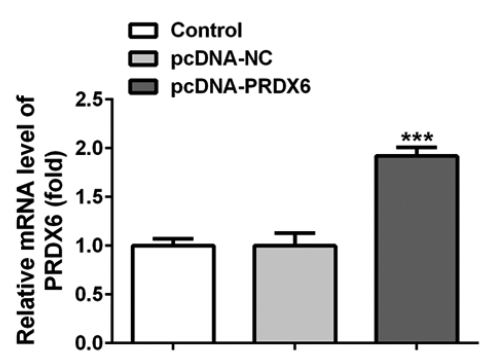

$\mathbf{E}$

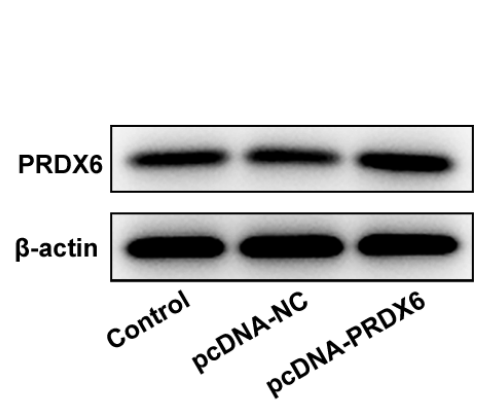

B
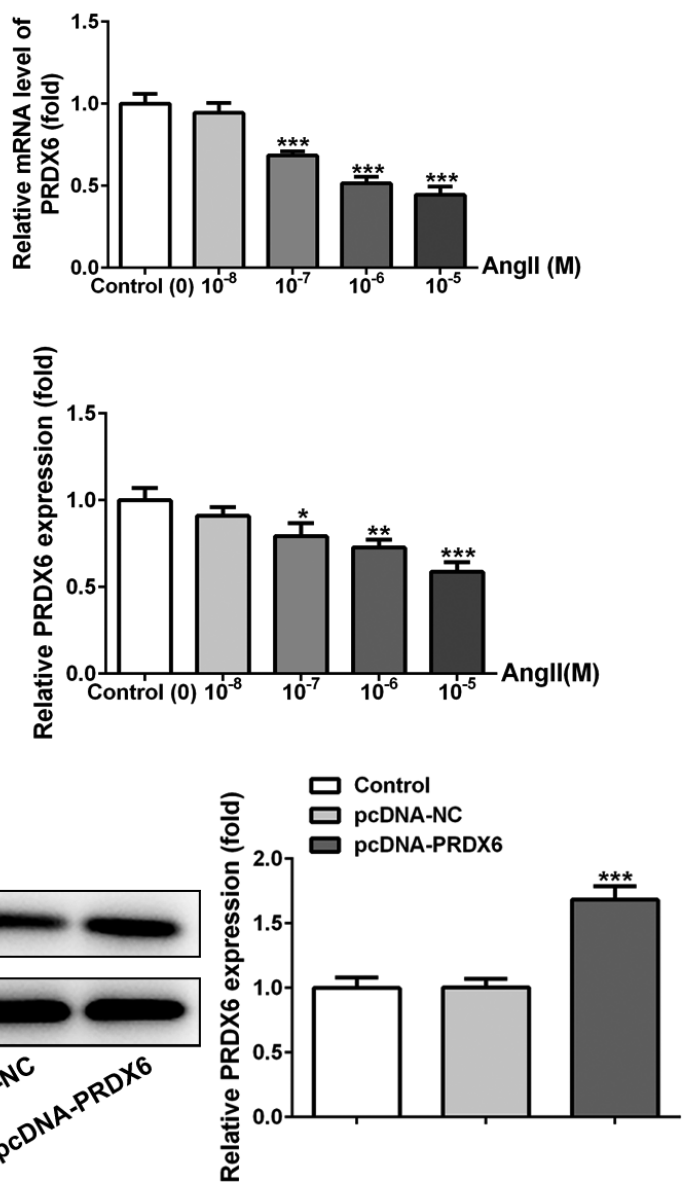

Figure 1. AngII-induced PRDX6 in Human umbilical vein endothelial cells (HUVECs) and transfection efficiency of overexpressed plasmids of pcDNA-PRDX6. A. AngII dose manner of cell viability of HUVECs was assessed using a CCK-8 assay. B. AngII dose manner of PRDX6 mRNA levels in HUVECs were determined using reverse transcriptase polymerase chain reaction RT-PCR. C. AngII dose manner of PRDX6 protein levels were measured by Western blot. D. and E. The mRNA and protein levels of PRDX6 were detected using RT-PCR and Western blot to test the transfection efficiency of pcDNA-PRDX6. Data are presented as the mean \pm SD $(n=3)$. ${ }^{\star} p<0.05$, ${ }^{* *} p<0.01,{ }^{* * *} p<0.001$ vs. Control group. 
A

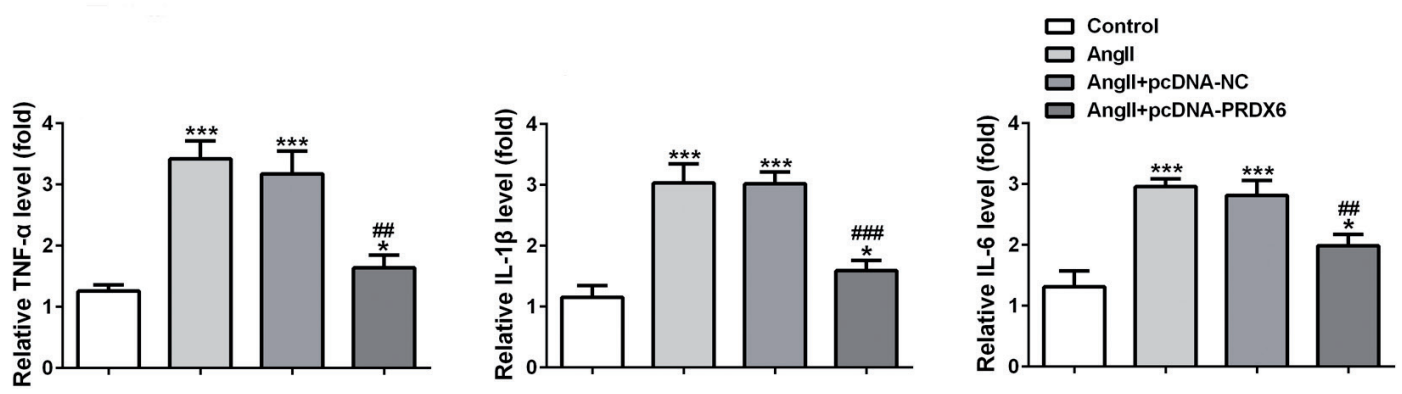

B

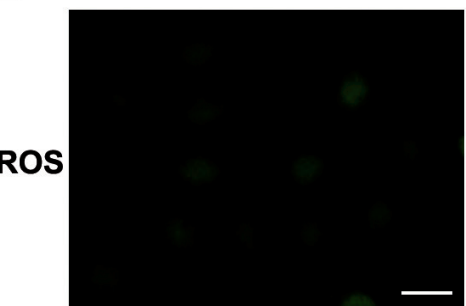

Control

C

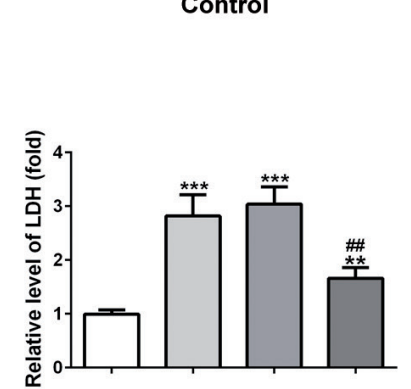

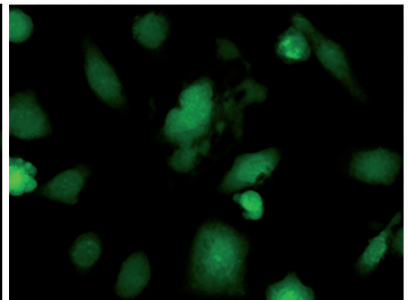

Angll

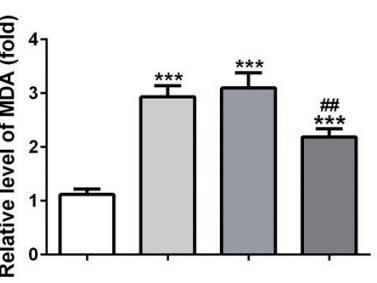

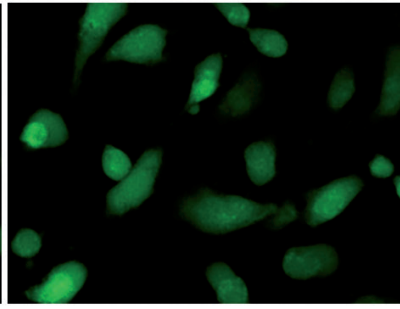

Angll+pcDNA-NC

D

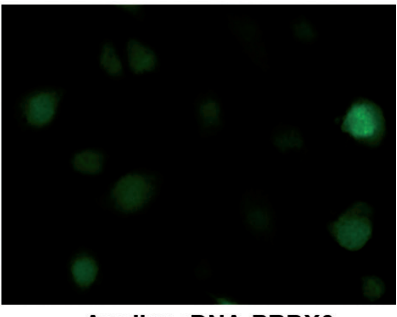

Angll+pcDNA-PRDX6

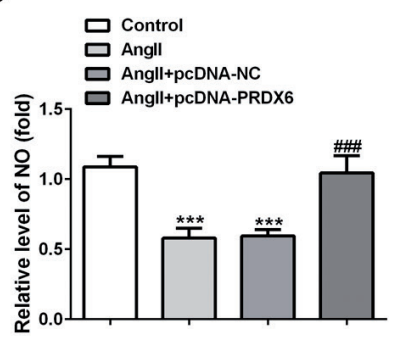

Figure 2. Effects of AngII and PRDX6 overexpression on inflammation and oxidative stress in HUVECs. A. Levels of TNF- $\alpha$, IL-6 and IL-1 $\beta$ in HUVECs after pcDNA-PRDX6 transfection were measured by ELISA. B. Cellular ROS accumulation was observed by microscopy following DCFH-DA staining in HUVECs after pcDNA-PRDX6 transfection. Magnification, $\times 100$; scale bar $=100 \mu \mathrm{m}$. C. The activity levels of LDH, MDA and ROS were determined by ELISA kits. D. NO production was assayed by Griess method using a nitrate/nitrite colorimetric assay kit in HUVECs after pcDNA-PRDX6 transfection. Data are presented as the mean $\pm \operatorname{SD}(n=3) .{ }^{\star} p<0.05,{ }^{* *} p<$ $0.01,{ }^{* * *} p<0.001$ vs. Control group; ${ }^{\# \#} p<0.01,{ }^{\# \# \#} p<0.001$ vs. AngII group.

treated HUVECs when compared with the control cells (Fig. 1B). Similar to RT-qPCR results, Western blot analysis showed that the protein expression of PRDX6 gradually decreased as the dose of AngII increased (Fig. 1C). These findings indicated that PRDX6 might participate in AngIIinduced cytotoxicity.

Based on the results, AngII of the trough value $\left(10^{-6} \mathrm{M}\right)$ accompanying by the $\geq 50 \%$ cell activity and a significant decrease in PRDX6 expression was selected for the following experimental study.

Overexpression of PRDX6 alleviates AngII-induced inflammatory and oxidative stress damage

The overexpression plasmid of PRDX6 was constructed and transfected into HUVECs. Results from RT-qPCR and
Western blot showed that pcDNA-PRDX6 vector remarkably upregulated the PRDX6 expression both in mRNA and protein levels (Fig. 1D and E). Subsequently, AngII-treated cells produced high levels of pro-inflammation factors (TNF- $\alpha$, IL-6 and IL-1 $\beta$ ), whereas overexpression of PRDX6 could suppress AngII-induced pro-inflammation factors production (Fig. 2A).

As presented in Fig. 2B, untreated HUVECs nearly failed to produce detectable levels of ROS, whereas AngII-treated cells produced high levels of ROS. Conversely, ROS expression was reduced in AngII-treated cells with pcDNA-PRDX6 transfection. As an important mediator of endothelial function maintenance, reduced levels of NO could accelerate the ED process. Furthermore, AngII could induce high levels of LDH, MDA, ROS in HUVECs, but a decrease of NO level (Fig. 2D). Interestingly, PRDX6 overexpression could notably 
reduce $\mathrm{LDH}, \mathrm{MDA}$, ROS levels, but elevated NO production (Fig. 2C and D). Overall, inflammatory and oxidative stress damage was associated with AngII treatment, which alleviated under PRDX6 overexpression.
Overexpression of PRDX6 recovers AngII-induced ED

eNOS (an ED marker) and SOD (antioxidant enzymes) have been reported to be involved in ED (Ripa et al. 2014).
A

C
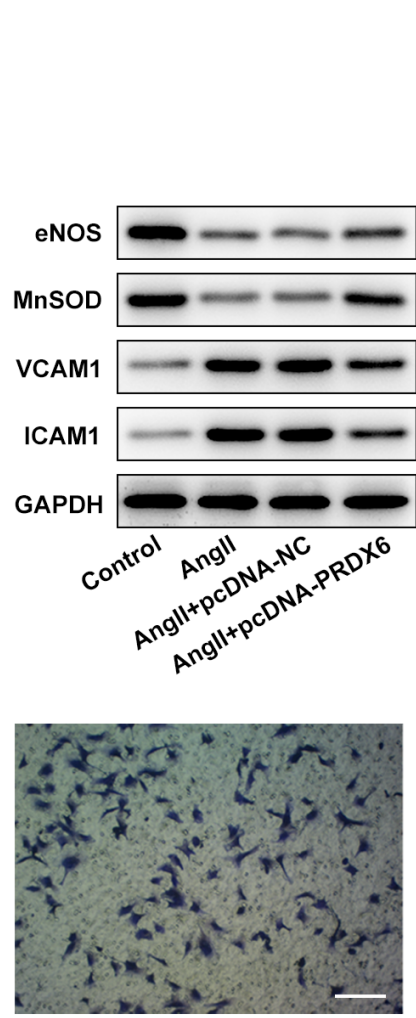

Control

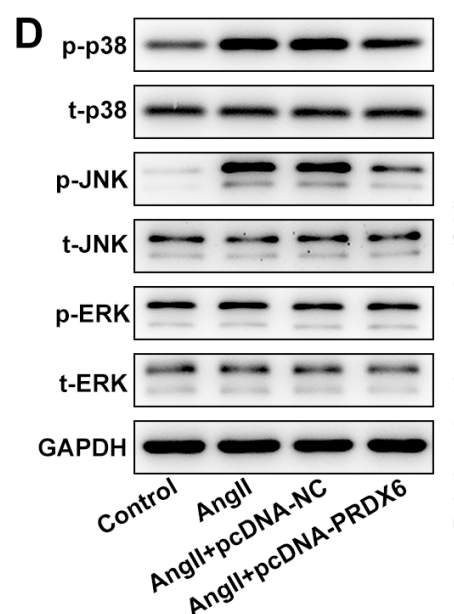

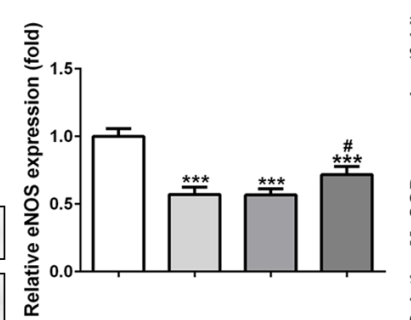
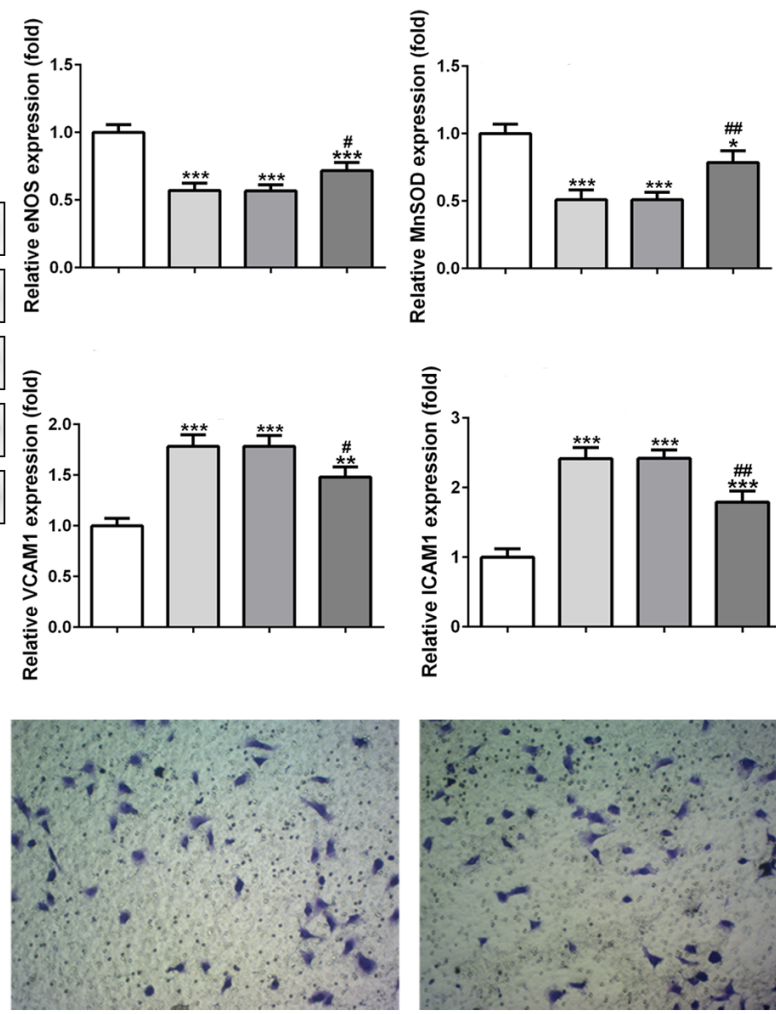

Angll
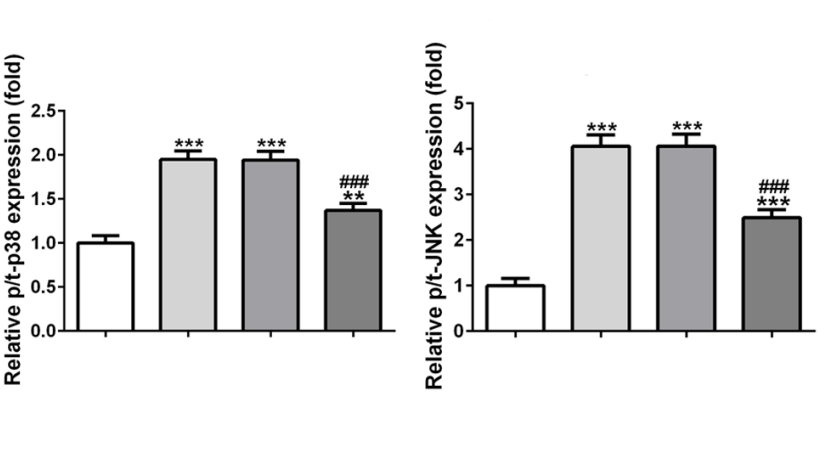
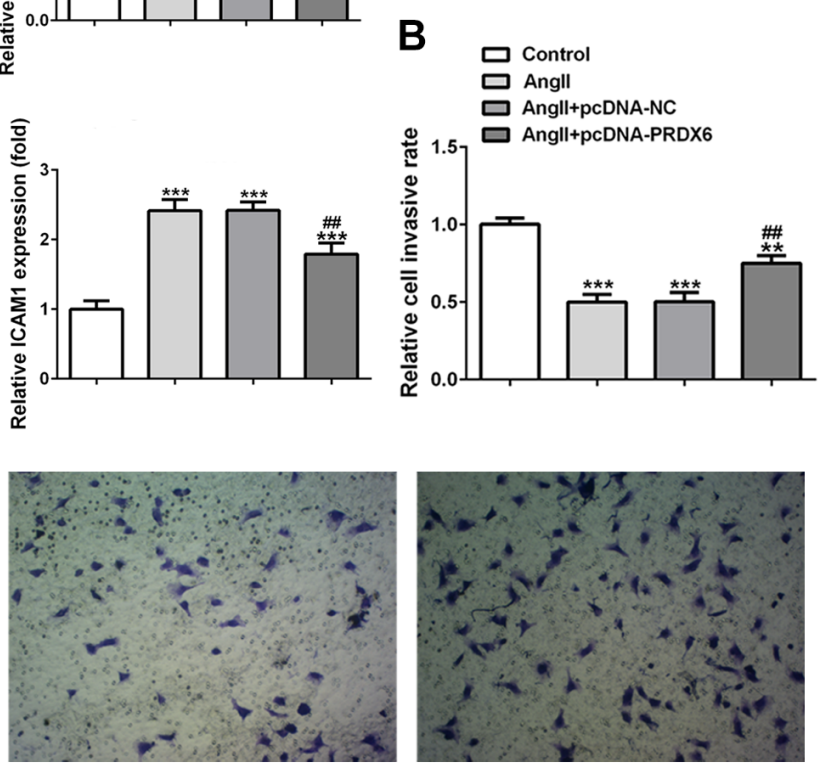

Angll+pcDNA-NC

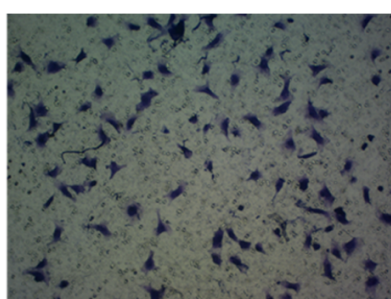

Angll+pcDNA-PRDX6

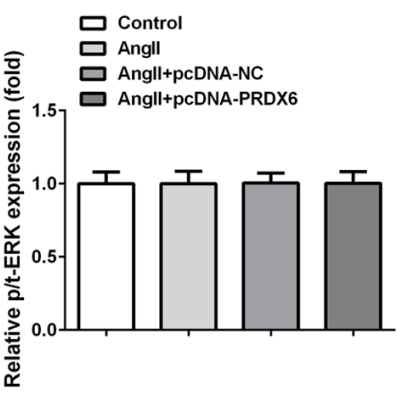

Figure 3. Overexpression of PRDX6 ameliorates AngII-induced endothelial dysfunction and migration inhibition. A. The protein expression levels of eNOS, MnSOD, VCAM1 and ICAM1 were determined by Western blotting. Blots were semi-quantified by densitometric analysis. B. and C. Statistical graph of data and representative pictures of Transwell assay for the detection of the migrated HUVECs after pcDNA-PRDX6 transfection. Magnification, $\times 100$; scale bar $=100 \mu \mathrm{m}$. D. The protein expression levels of p-p38 MAPK, p38 MAPK, p-JNK, JNK, p-ERK and ERK were determined by Western blot analysis. Blots were semi-quantified by densitometric analysis. Data are presented as the mean $\pm \mathrm{SD}(n=3) .{ }^{*} p<0.05,{ }^{* *} p<0.01,{ }^{* * *} p<0.001 v$ s. Control group; ${ }^{\#} p<0.05,{ }^{\# \#} p<0.01,{ }^{\# \# \#} p<0.001 v s$. AngII group. 
In the present study, AngII-treated HUVECs exhibited dramatic low expression of eNOS and MnSOD compared with untreated control cells. Additionally, HUVECs treated with pcDNA-PRDX6 vector after AngII exhibited increased eNOS and MnSOD expression compared with cells treated with AngII (Fig. 3A).

ICAM-1 and VCAM-1, belonging to inflammatory mediators, cause arterial dysfunction. The present results indicated that AngII could provoke ICAM-1 and VCAM-1 expression highly. Conversely, PRDX6 overexpression obviously suppressed the expression of ICAM-1 and VCAM-1 when compared with AngII-induced cells (Fig. 3A). These data indicated that AngII treatment may contribute to ED and overexpression of PRDX6 recovered AngII-induced ED in HUVECs.

Overexpression of PRDX6 restores AngII-induced the insufficient ability of HUVECs to invade and suppresses p38 MAPK, JNK expression

The Transwell migration assay showed that AngII exposure markedly inhibited the cell invasive rate of HUVECs. However, treatment with pcDNA-PRDX6 vector markedly enhanced AngII-induced insufficient migration of HUVECs (Fig. 3B and C).

To determine the effects of PRDX6 overexpression on downstream signaling pathways, we examined MAPK phosphorylation. Phospho (p)-p38 MAPK and p-JNK were significantly increased in AngII-induced HUVECs compared with control (Fig. 3D). These data indicate that increased inflammatory and oxidative stress damage in HUVECs is sufficient to activate downstream MAPK signaling pathways despite increased expression of vascular antioxidant enzymes. PRDX6-overexpressing significantly reduced p-p38 MAPK and p-JNK expression compared with AngII dispose (Fig. 3D). However, phospho-ERK and ERK levels were not significantly change always in in HUVECs (Fig. 3D), suggesting that PRDX6 dominate MAPK signal pathway in the vascular wall through regulating p38 MAPK and JNK expression.

PRDX6 inhibitor enhanced AngII-induced inflammatory and oxidative stress which was revered by $\mathrm{p} 38 \mathrm{MAPK}$ inhibitor or JNK inhibitor

Based on the related research result above, we speculate that PRDX6 knock down condition using PRDX6 inhibitor in HUVECs increased AngII-induced inflammatory, oxidative stress, ED and MAPK activation. As showed in Fig. 4A, predispose of PRDX6 inhibitor (MS, $20 \mathrm{uM}$ ) for $2 \mathrm{~h}$ aggravate the inflammation by upregulating TNF- $\alpha$, IL- 6 and IL-1 $\beta$ levels in AngII-induced HUVECs. Expectedly, MAPK suppression by p38 MAPK inhibitor (ML3404) or
JNK inhibitor (SP600125) sharply decreased TNF- $\alpha$, IL-6 and IL- $1 \beta$ levels compared to AngII+MS group (Fig. 4A). Similarly, treatment with MS, alongside AngII, resulted in increased levels of oxidative stress, including ROS, LDH, and MDA compared with in cells treated with AngII only. These findings indicate that PRDX6 could protect HUVECs against AngII-induced inflammatory and oxidative stress by suppressing MAPK signal pathway.

PRDX6 inhibitor enhanced AngII-induced ED and invasion inhibition which was revered by $\mathrm{p} 38$ MAPK inhibitor or JNK inhibitor

NO has anti-inflammatory properties, including inhibition of the inflammatory response in endothelial cells (Deanfield et al. 2007). In the present study, cells treated with MS and AngII had lower levels of NO, compared with MS-unstimulated AngII-treated cells (Fig. 4D). Notably, cells treated with either ML3404 or SP600125 alongside AngII exhibited improved NO production compared with MS- and AngII-treated cells (Fig. 4D). In addition, treatment with MS could suppress the expression of antioxidants eNOS and MnSOD, but induced ICAM-1 and VCAM-1 expression in AngII-induced cells. Rather, treatment with ML3404 had a similar effect to SP600125 against ED in MS-stimulated AngII-treated HUVECs through elevating eNOS and MnSOD and attenuating ICAM-1 and VCAM-1 expressions (Fig. 5A).These data indicate that antioxidants may mediate the effects of PRDX6 on AngII-induced ED.

MS exposure markedly inhibited cell invasion in AngIIinduced HUVECs (Fig. 5B and C). However, treatment with either ML3404 or SP600125 markedly enhanced cell invasion of HUVECs after MS + AngII stimulations (Fig. $5 B$ and 5C). These findings demonstrated that PRDX6 could protect HUVECs against AngII-induced ED and migration inhibition by suppressing MAPK signal pathway.

\section{Discussion}

There is always renin-angiotensin system (RAS) activation in patients with CVD. AngII is regarded as one of the important risk factors of accelerating atherosclerosis procedure. Many studies reported that AngII induced endothelial cell inflammation, oxidative stress, cell dysfunction and apoptosis (Bendall et al. 2007; Hu et al. 2017; Li et al. 2017), and participated in the local RAS in the artery vessels (Heeneman et al. 2007). AngII-induced endothelial injury is associated with atherosclerosis. In the present study, AngII could reduce PRDX6 expression in a dose-dependent manner in HUVECs, which indicated that PRDX6 may be involved in AngII-induced endothelial cell damage. 


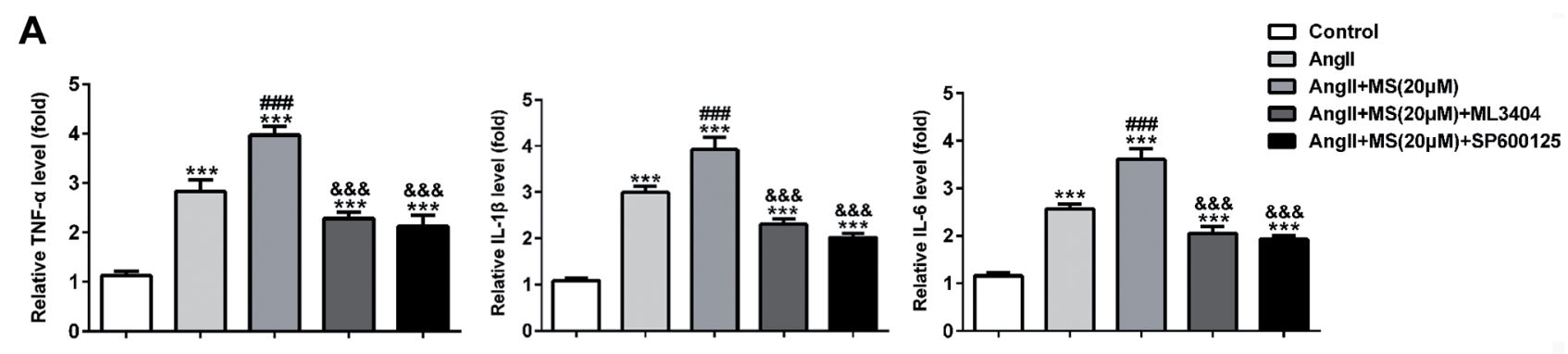

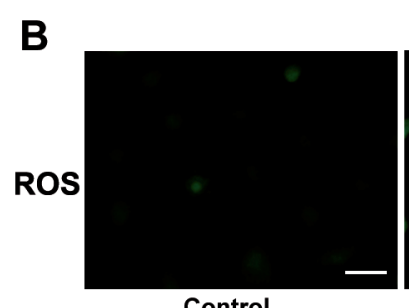

Control

C

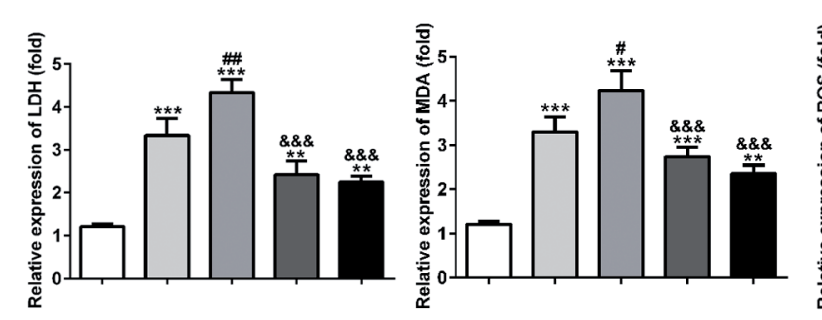

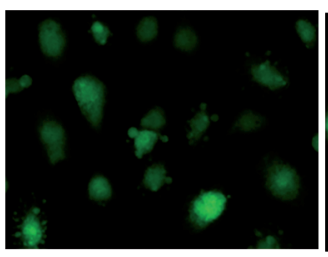

Angll

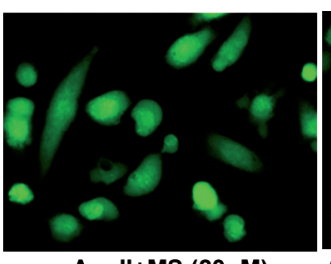

Angll+MS (20 $\mu \mathrm{M})$

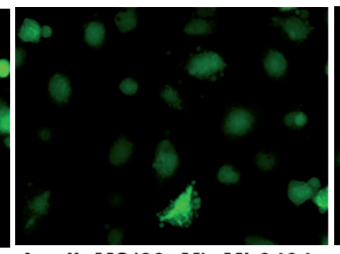

Angll+MS(20 $\mu M)+M L 3404$

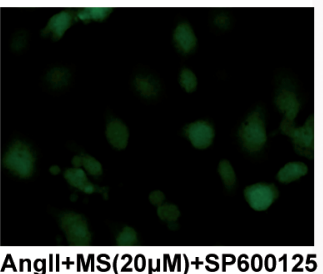

$\square$ Control

D $\square$ Angll

$\square$ Angll+Ms $(20 \mu \mathrm{M})$

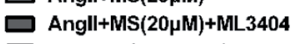

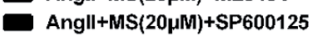
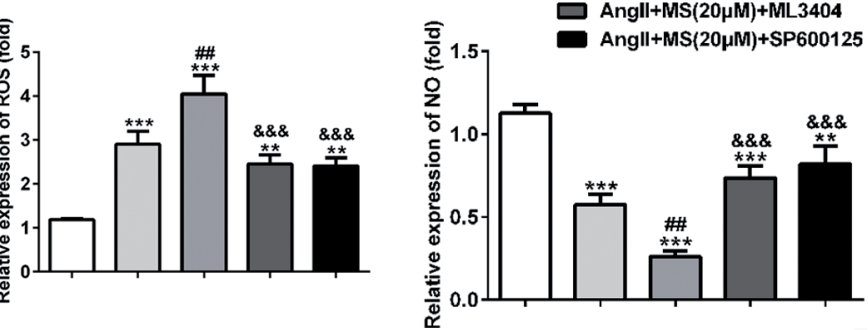

Figure 4. Effects of PRDX6 inhibitor and MAPK pathway inhibitor on inflammation and oxidative stress in AngII-induced HUVECs. A. Levels of TNF- $\alpha$, IL- 6 and IL-1 $\beta$ in HUVECs after MS treatment and the p38 MAPK inhibitor ML3404 or JNK inhibitor SP600125 were measured by ELISA. B. Cellular ROS accumulation was observed by microscopy following DCFH-DA staining in HUVECs. Magnification, $\times 100$; scale bar $=100 \mu \mathrm{m}$. C. The activity levels of LDH, MDA and ROS were determined by ELISA kits. D. NO production was assayed by Griess method using a nitrate/nitrite colorimetric assay kit in HUVECs. Data are presented as the mean \pm SD $(n=3)$. ${ }^{* *} p<0.01,{ }^{* * *} p<0.001$ vs. Control group; ${ }^{\#} p<0.05,{ }^{\# \#} p<0.01,{ }^{\# \# \#} p<0.001 v s$. AngII group; ${ }^{\& \& \&} p<0.001$ vs. the AngII + MS group. ML3404, the p38 MAPK inhibitor; SP600125, JNK inhibitor.

Inflammation status not only plays important functions in these conditions, but also cannot be ignored in the formation and advance of atherosclerosis and the occurrence of cardiovascular events. It has been demonstrated that AngII increases the expression of TNF- $\alpha$, MCP-1 and IL-8 in HUVECs (Jiang et al. 2007; Kim et al. 2008). In addition, atherosclerosis is closely related to inflammation (Libby et al. 2002). Our study demonstrated that PRDX6 overexpression could alleviate AngII-induced levels of TNF- $\alpha$, IL- $1 \beta$ and IL-6 remarkably in endothelia cells, suggesting PRDX6 has anti-inflammatory effect.

ROS is involved in different pathologies, including cardiovascular diseases as a therapeutic possibility (Paravicini and Touyz 2008). An excess of $\mathrm{O}_{2}{ }^{-}$may decrease $\mathrm{NO}$ availability, leading to endothelial dysfunction and a decrease in endothelium-dependent vasodilation. AngII is a strong stimulator of endothelial cell oxidative stress to induce the increase of endothelial ROS production, but ROS product superoxide anion could damage endothelial cell vitality (Deng et al. 2010). Many vascular disease states are associated with an increase in the production of ROS in the vessel wall, which is associated with reduced NO bioavailability, leading to endothelial dysfunction. Indeed, we observed an increase in the level of ROS, LDH and MDA, and a decrease of NO level in AngII-induced endothelia cells. And, surprisingly, PRDX6 overexpression could suppress oxidative stress and promote NO production. The present study hypothesized that PRDX6 exerts cytoprotective effects against AngIIinduced oxidative stress in HUVECs.

A previous study demonstrated that suppressed eNOS expression is associated with AngII cytotoxicity (Nakao et al. 2013). NO derived from eNOS is one of the critical signal 
molecules in the cardiovascular system. In addition to its vasodilator effect, NO also has anti-inflammatory properties, including inhibition of the inflammatory response in endothelial cells (Deanfield et al. 2007). In the atherosclerotic process, ROS can promote oxidized LDL (oxLDL) formation, stimulate matrix metalloproteinases (MMPs), improve vascular smooth muscle cell (VSMC) growth and provoke inflammatory mediator production, including ICAM-1 and VCAM-1. Together with the present findings, AngII stimulation caused a decrease in the protein expression of eNOS and MnSOD as well as the cell migration in endothelial cells, but improved VSMC growth. These results suggested that AngII may result in HUVECs dysfunction. Overexpression of PRDX6 induced an increase in the protein levels of MnSOD and eNOS, both of which may contribute to the maintenance of normal basal blood pressure and endothelium-dependent vasorelaxation through enhanced $\mathrm{O}_{2}{ }^{-\cdot}$ removal and $\mathrm{NO}$ production. Additionally, PRDX6 could also improve the
ED by inhibiting ICAM-1 and VCAM-1 expression, and augmenting cell migration.

MAPK pathway is related to AngII-activated production of inflammatory factors in HUVECs (Deng et al. 2015). ROS generation is stimulated by $\kappa$ opioid receptor (KOR) activation through phosphorylated c-Jun N-terminal kinase and inhibited by p38 MAPK activation (Schattauer et al. 2019). MAPK signal pathway consists of kinases that are activated one after another, for example, p38, JNK and ERK. In our data, AngII-induced HUVECs showed increased p-JNK and p-p38 MAPK activation is compared to control cells. Overexpression of PRDX6 suppressed p-JNK and p-p38 MAPK expression, but has no change on the ERK expression. A previous study indicated that PRDX6 overexpression inactivates p38 MAPK and JNK pathway by reducing LPS-induced ROS level in the kidney, resulting in inhibition of renal apoptosis and leukocyte infiltration, and led to attenuation of LPSinduced acute kidney injury (Lee et al. 2017). Similar to our
A

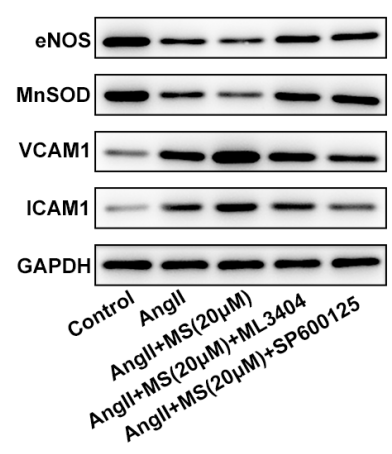

C

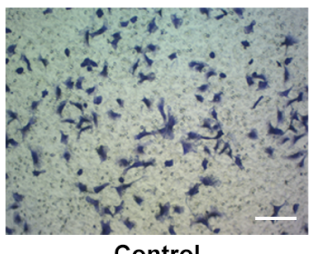

Control
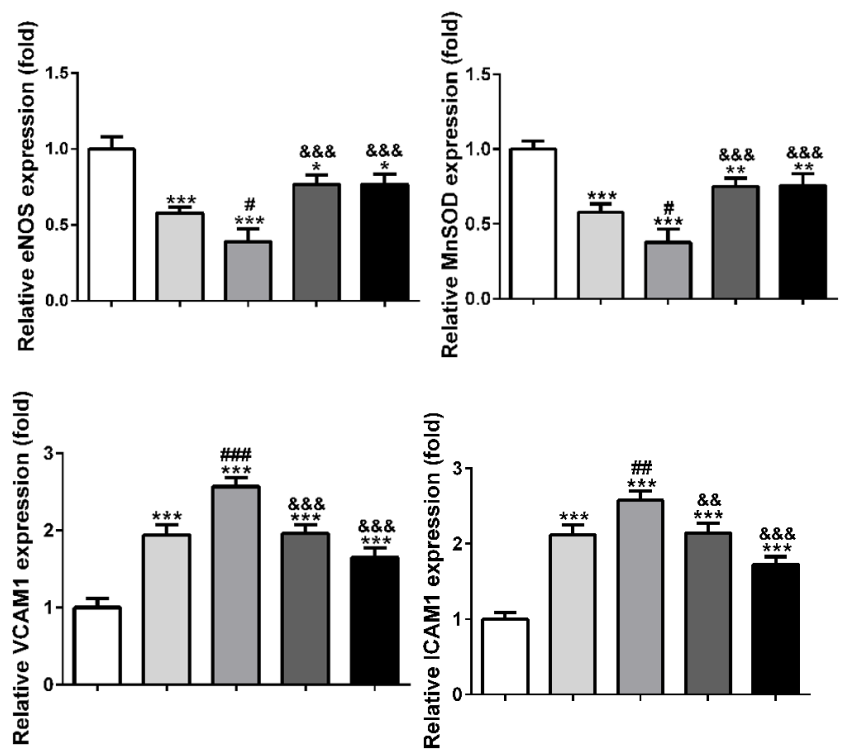

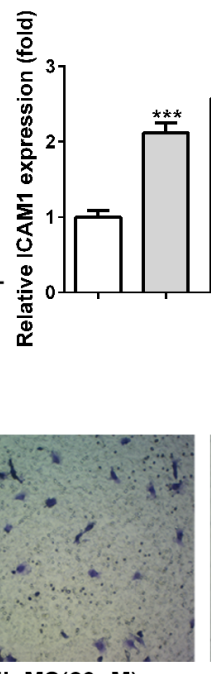

Angll+MS $(20 \mu \mathrm{M})$

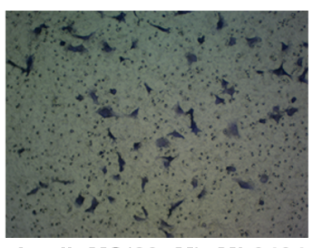

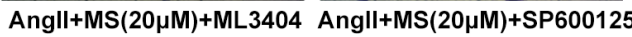

Figure 5. Effects of PRDX6 inhibitor and MAPK pathway inhibitor on AngII-induced endothelial dysfunction and migration in AngII-induced HUVECs. A. The protein expression levels of eNOS, MnSOD, VCAM1 and ICAM1 were determined by Western blotting. Blots were semi-quantified by densitometric analysis. B. and C. Statistical graphs of data and representative pictures of Transwell assay for the detection of the migrated HUVECs after pcDNA-PRDX6 transfection. Magnification, $\times 100 ;$ scale bar $=$ $100 \mu \mathrm{m}$. Data are presented as the mean $\pm \mathrm{SD}(n=3) .{ }^{*} p<0.05,{ }^{* *} p<0.01,{ }^{* * *} p<0.001$ vs. Control group; ${ }^{\#} p<0.05,{ }^{\# \#} p<$ $0.01,{ }^{\# \#} p<0.001 v s$. AngII group; ${ }^{\& \&} p<0.01 \& \& \& p<0.001$ vs. AngII+MS group. ML3404, the p38 MAPK inhibitor; SP600125, JNK inhibitor. 
results, we came to the conclusion that PRDX6 inactivates p38 MAPK/JNK pathway by reducing the AngII-induced ROS concentration in HUVECs, resulting in inhibition of inflammation and oxidative stress and led to attenuation of AngII-induced endothelial cell damage.

PRDX6 is expressed in almost all mammalian tissues and is highly expressed in solid organs. PRDX6 silencing markedly suppressed colorectal cancer (CRC) cell migration and invasiveness while also inducing cell cycle arrest as well as the generation of ROS; specific overexpression of PRDX6 had the opposite effect (Huang et al. 2018). PRDX6 is more important than other antioxidants under excessive oxidative stress in vivo (Wang et al. 2003). It has been reported that downregulated expression of PRDX6 decreased the survival rates, and increased apoptosis and the level of MDA and ROS in HCECs under UV-B irradiation (Yu et al. 2019). Herein, we found that the PRDX6 inhibitor obviously aggravated inflammation, oxidative stress and ED, and increased the inhibitory effect of AngII on cell migration of HUVECs. Conventional KOR agonists stimulate ROS generation through a JNK-PRDX6 pathway (Schattauer et al. 2019). In this study, the p38 MAPK inhibitor or JNK inhibitor were used to inhibit the AngII + MS-activated MAPK signaling pathway to verify that MAPK signaling pathway was involved in the PRDX6's protective effect on endothelial cell dysfunction. The present investigation demonstrated that under the condition of that MAPK signaling pathway was restrained, the levels of inflammation, oxidative stress and the degree of cell dysfunction caused by AngII and MS are greatly improved, which provided a molecular mechanism demonstrating the role of PRDX6 in mediating vascular inflammation, oxidative stress and ED in AngII-induced endotheliocyte.

\section{Conclusion}

The present study hypothesized that PRDX6 exerts cytoprotective effects against AngII-induced ED in HUVECs via the inhibition of MAPK signaling pathway. ED is predictive of cardiovascular events and long-term clinical outcome. Therefore, PRDX6 can be used to guide the clinical treatment of CVD and develop new targeted drugs.

Conflict of interest. The authors declared they do not have anything to disclose regarding conflict of interest with respect to this manuscript.

\section{References}

Bendall JK, Rinze R, Adlam D, Tatham AL, de Bono J, Wilson N, Volpi E, Channon KM (2007): Endothelial Nox2 overexpression potentiates vascular oxidative stress and hemodynamic response to angiotensin II: studies in endothelial-targeted Nox2 transgenic mice. Circ. Res. 100, 1016-1025 https://doi.org/10.1161/01.RES.0000263381.83835.7b

Cai H (2005): Hydrogen peroxide regulation of endothelial function: origins, mechanisms, and consequences. Cardiovasc. Res. 68, 26-36

https://doi.org/10.1016/j.cardiores.2005.06.021

Cai H, Harrison DG (2000): Endothelial dysfunction in cardiovascular diseases: the role of oxidant stress. Circ. Res. 87, 840-844 https://doi.org/10.1161/01.RES.87.10.840

Chatterjee S, Feinstein SI, Dodia C, Sorokina E, Lien YC, Nguyen S, Debolt K, Speicher D, Fisher AB (2011): Peroxiredoxin 6 phosphorylation and subsequent phospholipase A2 activity are required for agonist-mediated activation of NADPH oxidase in mouse pulmonary microvascular endothelium and alveolar macrophages. J. Biol. Chem. 286, 11696-11706 https://doi.org/10.1074/jbc.M110.206623

Deanfield JE, Halcox JP, Rabelink TJ (2007): Endothelial function and dysfunction: testing and clinical relevance. Circulation 115, 1285-1295 https://doi.org/10.1161/CIRCULATIONAHA.106.652859

Deng B, Fang F, Yang T, Yu Z, Zhang B, Xie X (2015): Ghrelin inhibits AngII -induced expression of TNF-alpha, IL-8, MCP1 in human umbilical vein endothelial cells. Int. J. Clin. Exp. Med. 8, 579-588 https://doi.org/10.1097/MD.0000000000000579

Deng B, Fang L, Chen X, Chen M, Xie X (2010): Effect of ghrelin on angiotensin II induced human umbilicus vein endothelial cell oxidative stress and endothelial cell injury. Zhong Nan Da Xue Xue Bao Yi Xue Ban 35, 1037-1047 (in Chinese)

Harrison DG, Widder J, Grumbach I, Chen W, Weber M, Searles C (2006): Endothelial mechanotransduction, nitric oxide and vascular inflammation. J. Intern. Med. 259, 351-363 https://doi.org/10.1111/j.1365-2796.2006.01621.x

Heeneman S, Sluimer JC, Daemen MJ (2007): Angiotensin-converting enzyme and vascular remodeling. Circ. Res. 101, 441-454 https://doi.org/10.1161/CIRCRESAHA.107.148338

Hu HJ, Jiang ZS, Qiu J, Zhou SH, Liu QM (2017): Protective effects of hydrogen sulfide against angiotensin II-induced endoplasmic reticulum stress in HUVECs. Mol. Med. Rep. 15, 2213-2222 https://doi.org/10.3892/mmr.2017.6238

Huang WS, Huang CY, Hsieh MC, Kuo YH, Tung SY, Shen CH, Hsieh YY, Teng CC, Lee KC, Lee KF, Kuo HC (2018): Expression of PRDX6 correlates with migration and invasiveness of colorectal cancer cells. Cell Physiol. Biochem. 51, 2616-2630 https://doi.org/10.1159/000495934

Jiang XY, Gao GD, Zhou J, Guo R, Li YX (2007): Role of AT2 receptors on angiotensin II-induced tumor necrosis factor alpha and interleukin 1 beta secretion in adult rat cardiac fibroblasts. Nan Fang Yi Ke Da Xue Xue Bao 27, 1307-1309 (in Chinese)

Kim HY, Kang YJ, Song IH, Choi HC, Kim HS (2008): Upregulation of interleukin-8/CXCL8 in vascular smooth muscle cells from spontaneously hypertensive rats. Hypertens. Res. 31, 515-523 https://doi.org/10.1291/hypres.31.515

Lee DH, Park JH, Han SB, Yoon DY, Jung YY, Hong JT (2017): Peroxiredoxin 6 overexpression attenuates lipopolysaccharideinduced acute kidney injury. Oncotarget 8, 51096-51107 https://doi.org/10.18632/oncotarget.17002 
Li M, Liu X, He Y, Zheng Q, Wang M, Wu Y, Zhang Y, Wang C (2017): Celastrol attenuates angiotensin II mediated human umbilical vein endothelial cells damage through activation of Nrf2/ERK1/2/Nox2 signal pathway. Eur. J. Pharmacol. 797, 124-133 https://doi.org/10.1016/j.ejphar.2017.01.027

Libby P, Ridker PM, Maseri A (2002): Inflammation and atherosclerosis. Circulation 105, 1135-1143 https://doi.org/10.1161/hc0902.104353

Mueller CF, Laude K, McNally JS, Harrison DG (2005): ATVB in focus: redox mechanisms in blood vessels. Arterioscler. Thromb. Vasc. Biol. 25, 274-278 https://doi.org/10.1161/01.ATV.0000149143.04821.eb

Nakao T, Morita H, Maemura K, Amiya E, Inajima T, Saito Y, Watanabe M, Manabe I, Kurabayashi M, Nagai R, Komuro I (2013): Melatonin ameliorates angiotensin II-induced vascular endothelial damage via its antioxidative properties. J. Pineal. Res. 55, 287-293 https://doi.org/10.1111/jpi.12071

Nathan C (2003): Specificity of a third kind: reactive oxygen and nitrogen intermediates in cell signaling. J. Clin. Invest. 111, 769-778 https://doi.org/10.1172/JCI200318174

Paravicini TM, Touyz RM (2008): NADPH oxidases, reactive oxygen species, and hypertension: clinical implications and therapeutic possibilities. Diabetes Care 31, S170-180 https://doi.org/10.2337/dc08-s247

Patel P, Chatterjee S (2019): Peroxiredoxin 6 in endothelial signaling. Antioxidants (Basel) 8, 63 https://doi.org/10.3390/antiox8030063

Rhee SG, Chae HZ, Kim K (2005): Peroxiredoxins: a historical overview and speculative preview of novel mechanisms and emerging concepts in cell signaling. Free Radic. Biol. Med. 38, 1543-1552 https://doi.org/10.1016/j.freeradbiomed.2005.02.026

Rhee SG, Kang SW, Chang TS, Jeong W, Kim K (2001): Peroxiredoxin, a novel family of peroxidases. IUBMB Life 52, 35-41 https://doi.org/10.1080/15216540252774748

Ripa P, Ornello R, Pistoia F, Carolei A, Sacco S (2014): The renin-angiotensin system: a possible contributor to migraine pathogenesis and prophylaxis. Expert Rev. Neurother. 14, 1043-1055 https://doi.org/10.1586/14737175.2014.946408

Schattauer SS, Bedini A, Summers F, Reilly-Treat A, Andrews MM, Land BB, Chavkin C (2019): Reactive oxygen species (ROS) generation is stimulated by kappa opioid receptor activation through phosphorylated c-Jun N-terminal kinase and inhibited by $\mathrm{p} 38$ mitogen-activated protein kinase (MAPK) activation. J. Biol. Chem. 294, 16884-16896

https://doi.org/10.1074/jbc.RA119.009592

Shishehbor MH, Aviles RJ, Brennan ML, Fu X, Goormastic M, Pearce GL, Gokce N, Keaney JF, Jr., Penn MS, Sprecher DL, Vita JA, Hazen SL (2003): Association of nitrotyrosine levels with cardiovascular disease and modulation by statin therapy. JAMA 289, 1675-1680 https://doi.org/10.1001/jama.289.13.1675

Sun M, Huang X, Yan Y, Chen J, Wang Z, Xie M, Li J (2012): Rac1 is a possible link between obesity and oxidative stress in Chinese overweight adolescents. Obesity (Silver Spring) 20, 2233-2240 https://doi.org/10.1038/oby.2012.63

Touyz RM (2005): Molecular and cellular mechanisms in vascular injury in hypertension: role of angiotensin II. Curr. Opin. Nephrol. Hypertens. 14, 125-131 https://doi.org/10.1097/00041552-200503000-00007

Vazquez-Medina JP, Dodia C, Weng L, Mesaros C, Blair IA, Feinstein SI, Chatterjee S, Fisher AB (2016): The phospholipase A2 activity of peroxiredoxin 6 modulates NADPH oxidase 2 activation via lysophosphatidic acid receptor signaling in the pulmonary endothelium and alveolar macrophages. FASEB J. 30, 2885-2898 https://doi.org/10.1096/fj.201500146R

Wang X, Phelan SA, Forsman-Semb K, Taylor EF, Petros C, Brown A, Lerner CP, Paigen B (2003): Mice with targeted mutation of peroxiredoxin 6 develop normally but are susceptible to oxidative stress. J. Biol. Chem. 278, 25179-25190 https://doi.org/10.1074/jbc.M302706200

Wang Y, Feinstein SI, Fisher AB (2008): Peroxiredoxin 6 as an antioxidant enzyme: protection of lung alveolar epithelial type II cells from $\mathrm{H} 2 \mathrm{O} 2$-induced oxidative stress. J. Cell Biochem. 104, 1274-1285 https://doi.org/10.1002/jcb.21703

Yu H, Yu Y, Zhao Z, Cui L, Hou J, Shi H (2019): Prdx6 is required to protect human corneal epithelial cells against ultraviolet B injury. Eur. J. Ophthalmol. 2019, 1120672119896426 https://doi.org/10.1177/1120672119896426

Received: April 30, 2020

Final version accepted: July 17, 2020 